\title{
Universiteit
}

Leiden

The Netherlands

\section{Adiabatic quantization of Andreev quantum billiard levels}

Beenakker, C.W.J.; Silvestrov, P.G.; Goorden, M.C.

\section{Citation}

Beenakker, C. W. J., Silvestrov, P. G., \& Goorden, M. C. (2003). Adiabatic quantization of Andreev quantum billiard levels. Retrieved from https://hdl.handle.net/1887/1279

Version: $\quad$ Not Applicable (or Unknown)

License: $\quad$ Leiden University Non-exclusive license

Downloaded from: https://hdl.handle.net/1887/1279

Note: To cite this publication please use the final published version (if applicable). 


\title{
Adiabatic Quantization of Andreev Quantum Billiard Levels
}

\author{
P G Silvestiov, ${ }^{12}$ M C Goorden, ${ }^{1}$ and C W J Beenakker ${ }^{1}$ \\ 'Instituut-Lorentz. Unvversiteit Leiden, PO Box 95062300 RA Leiden, The Netherlands \\ ${ }^{2}$ Budker Instutute of Nuclear Physics 630090 Novosiburk Russta
}

(Received 12 August 2002, published 17 March 2003)

\begin{abstract}
We identify the time $T$ between Andieev ieflections as a classical adiabatic invaisant in a ballistic chaotic cavity (Lyapunov exponent $\lambda$ ), coupled to a supeiconductor by an $N$ mode constriction Quantization of the adiabatically invariant tor us in phase space gives a discrete set of periods $T_{n}$, which in turn generate a ladder of excited states $\varepsilon_{n m}=(m+1 / 2) \pi \hbar / T_{n}$ The laigest quantized pei lod is the Ehrenfest time $T_{0}=\lambda^{-1} \ln N$ Piojection of the invariant toi us onto the coordinate plane shows that the wave functions inside the cavity are squeezed to a transverse dimension $W / \sqrt{N}$, much below the width $W$ of the constiction
\end{abstract}

DOI 10 1103/PhysRevLett 90116801

The notion that quantized energy levels may be associated with classical adiabatic invairants goes back to Ehienfest and the bit th of quantum mechanics [1] It was successful in providing a semiclassical quantization scheme for special integiable dynamical systems but falled to descirbe the generic nonıntegiable case Adrabatic invaliants play an interesting but minor role in the quantization of chaotic systems $[2,3]$

Since the existence of an adiabatic invaisant is the exception rather than the 1 ule, the emergence of a new one quite often teaches us something useful about the system An example fiom condensed matter physics is the quantum Hall effect, in which the semiclassical theory is based on two adıabatic invariants the flux thiough a cyclotion orbit and the flux enclosed by the orbit center as it slowly diffts along an equipotential [4] The stiong magnetic field suppiesses chaotic dynamics in a smooth potential landscape, rendering the motion quasi-integrable

Some time ago it was realized that Andieev reflection has a similar effect on the chaotic motion in an electron billiard coupled to a superconductor [5] An election trajectory is retiaced by the hole that is produced upon absorption of a Cooper pail by the superconductor At the Fermi energy $E_{F}$ the dynamics of the hole is piecisely the time reverse of the election dynamics, so that the motion is strictly periodic The peilod fiom election to hole and back to election is twice the time $T$ between Andieev reflections For finite excitation eneigy $\varepsilon$ the election (at energy $E_{F}+\varepsilon$ ) and the hole (at eneigy $E_{F}-\varepsilon$ ) follow slightly different trajectories, so the or bit does not quite close and dilfts around in phase space This $d_{11} f t$ has been studied in a valiety of contexts [5-9] but not in connection with adiabatic invailants and the associated quantization conditions It is the puipose of this Letter to make that connection and point out a stilking physical consequence The wave functions of Andieev levels fill the cavity in a highly nonuniform "squeezed" way, which has no counterpat in normal
PACS numbers $7363 \mathrm{Kv}, 0545 \mathrm{Mt}, 7450+\mathrm{r} 7445+\mathrm{c}$

state chaotic or regular billiards In particula1, the squeezing is distinct fiom periodic orbit scailing [10] and entriely different fiom the random supesposition of plane waves expected for a fully chaotic billiard [11]

Adiabatic quantization bieaks down near the excitation gap, and we will argue that iandom-matrix theor y [12] can be used to quantize the lowest-lying excitations above the gap This will lead us to a formula for the gap that crosses over from the Thouless energy to the inverse Ehienfest time as the number of modes in the point contact is increased

To illustiate the problem we represent in Figs 1 and 2 the quasiper ıdic motion in a paitıculaı Andieev billiard (It is similar to a Sinat billaard but has a smooth potentral $V$ in the interior to favor adrabaticity) Figuie 1 shows a trajectory in real space while Fig 2 is a section of phase space at the interface with the superconductor $(y=0)$ The tangential component $p_{\lambda}$ of the election momentum 1s plotted as a function of the coordinate $\lambda$ along the interface Each point in thıs Porncaré map corlesponds to one collision of an election with the interface (The collisions of holes are not plotted) The election is retioieflected as a hole with the same $p_{\lambda}$ At $\varepsilon=0$ the component $p$, is also the same, and so the hole retraces the path of the election (the hole velocity being opposite to its momentum) At nonzero $\varepsilon$ the 1etroreflection occurs with a slight change in $p_{\text {, }}$, because of the difference $2 \varepsilon$ in the kinetic energy of elections and holes The resulting slow dirft of the periodic trajectory traces out a contour in the surface of section The adiabatic invaliant is the function of $x, p_{2}$ that is constant on the contour We have found numerically that the dift follows isochronous contours $C_{T}$ of constant time $T\left(x, p_{\lambda}\right)$ between Andieev reflections [13] Let us now demonstrate analytically that $T$ is an adiabatic invai iant

We conside1 the Poincaié map $C_{T} \rightarrow C(\varepsilon, T)$ at energy $\varepsilon$ If $\varepsilon=0$ the Poincaie map is the identity, so $C(0 T)=C_{T}$ For adrabatic invatiance we need to prove that $\lim _{\varepsilon \rightarrow 0} d C / d \varepsilon=0$, so that the difference between 


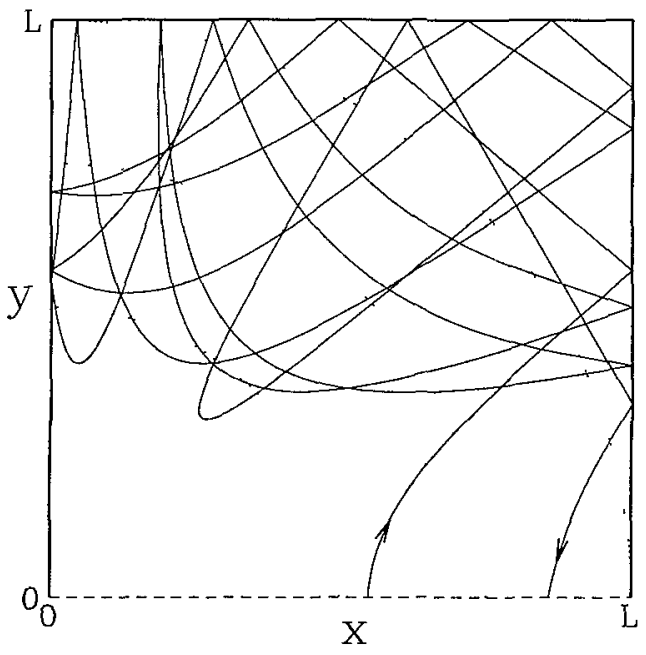

FIG. 1. Classical trajectory in an Andreev billiard. Particles in a two-dimensional electron gas are deflected by the potential $V=\left[1-(r / L)^{2}\right] V_{0}$ for $r<L, V=0$ for $r>L$. (The dotted circles are equipotent1als.) There is specular reflection at the boundaries with an insulator (thick solid lines) and Andreev reflection at the boundary with a superconductor (dashed line). The tiajectory follows the motion between two Andreev reflections of an electron near the Fermi energy $E_{F}=0.84 V_{0}$. The Andreev reflected hole retraces this trajectory in the opposite direction.

$C(\varepsilon, T)$ and $C_{T}$ is of higher order than $\varepsilon$ [14]. Since the contour $C(\varepsilon, T)$ can be locally represented by a function $p_{x}(x, \varepsilon)$, we need to prove that $\lim _{\varepsilon \rightarrow 0} \partial p_{\imath}(x, \varepsilon) / \partial \varepsilon=0$.

In order to prove this, it is convenient to decompose the map $C_{T} \rightarrow C(\varepsilon, T)$ into three separate stages, starting out as an electron (from $C_{T}$ to $C_{+}$), followed by Andreev reflection $\left(C_{+} \rightarrow C_{-}\right)$, and then concluded as a hole [from $C_{\text {- }}$ to $C(\varepsilon, T)$ ]. Andreev reflection introduces a discontinuity in $p_{y}$ but leaves $p_{x}$ unchanged, so $C_{+}=$ $C_{\text {_. }}$. The flow in phase space as electron $(+)$ or hole $(-)$ at energy $\varepsilon$ is described by the action $S_{+}(\mathbf{q}, \varepsilon)$, such that $\mathbf{p}^{ \pm}(\mathbf{q}, \varepsilon)=\partial S_{ \pm} / \partial \mathbf{q}$ gives the local dependence of (electron or hole) momentum $\mathbf{p}=\left(p_{\lambda}, p_{y}\right)$ on position $\mathbf{q}=$ $(x, y)$. The derivative $\partial S_{ \pm} / \partial \varepsilon=t_{ \pm}(\mathbf{q}, \varepsilon)$ is the time elapsed since the previous Andreev reflection. Since by construction $t_{ \pm}(x, y=0, \varepsilon=0)=T$ is independent of the position $x$ of the end of the trajectory, we find that $\lim _{\varepsilon \rightarrow 0} \partial p_{x}^{ \pm}(x, y=0, \varepsilon) / \partial \varepsilon=0$, completing the proof.

The drift $\left(\delta x, \delta p_{x}\right)$ of a point in the Poincare map is perpendicular to the vector $\left(\partial T / \partial x, \partial T / \partial p_{x}\right)$. Using also that the map is area preserving, it follows that

$$
\left(\delta x, \delta p_{\imath}\right)=\varepsilon f(T)\left(\partial T / \partial p_{\lambda},-\partial T / \partial x\right)+O\left(\varepsilon^{2}\right),
$$

with a prefactor $f(T)$ that is the same along the entire contour.

The adiabatic invariance of isochronous contours may alter natively be obtained from the adiabatic invariance of the action integral $I$ over the quasiperiodic motion from

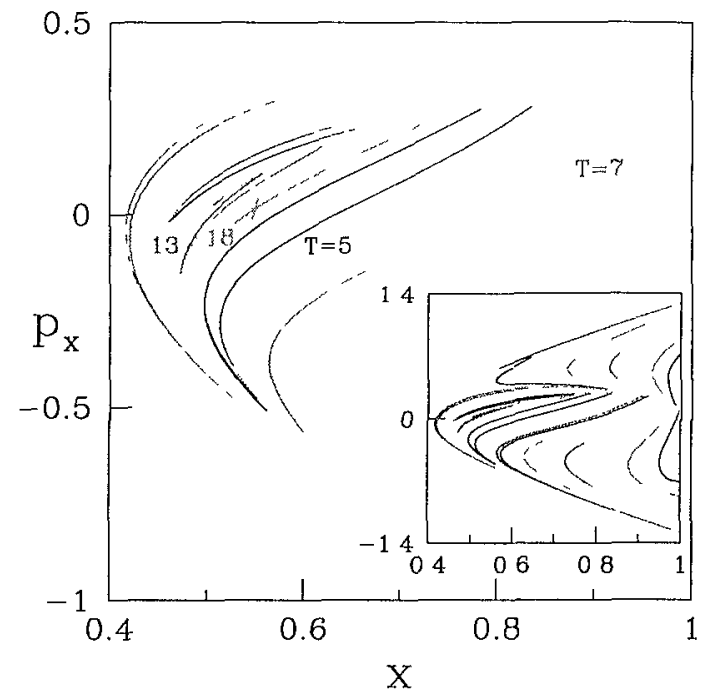

FIG. 2 (color online). Poncaré map for the Andreev billiand of Fig. 1. Each dot represents a starting point of an electron trajectory, at position $x$ (1n units of $L$ ) along the interface $y=0$ and with tangentral momentum $p_{\imath}$ (in units of $\sqrt{m V_{0}}$ ). The inset shows the full surface of the scction, while the main plot is an enlargement of the central region. The $\mathrm{d}_{1}$ ifting quasiper1odic motion follows contours of constant time $T$ between Andreev reflections. The cross marks the starting point of the trajectory shown in the previous figure, having $T=18$ (in units of $\sqrt{m L^{2} / V_{0}}$ ).

electron to hole and back to electron:

$$
I=\oint p d q=\varepsilon \oint \frac{d q}{q}=2 \varepsilon T .
$$

Since $\varepsilon$ is a constant of the motion, adiabatic invariance of $I$ implies adiabatic invariance of the time $T$ between Andreev reflections. This is the way in which adiabatic invariance is usually proven in textbooks. Our proof explicitly takes into account the fact that phase space in the Andreev billiard consists of two sheets, joined in the constriction at the interface with the superconductor, with a discontinuity in the action on going from one sheet to the other.

The contours of large $T$ enclose a very small area. This will play a crucial role when we quantize the billiard, so let us estimate the area. It is convenient for this estimate to measure $p_{2}$ and $x$ in units of the Fermi momentum $p_{F}$ and width $W$ of the constriction to the superconductor. The highly elongated shape evident in Fig. 2 is a consequence of the exponential divergence in time of nearby trajectories, characteristic of chaotic dynamics. The rate of divergence is the Lyapunov exponent $\lambda$. (We consider a fully chaotic phase space.) Since the Hamiltonian flow is a rea preserving, a stretching $\ell_{+}(t)=$ $\ell_{+}(0) e^{\lambda t}$ of the dimension in one direction needs to be compensated by a squeezing $\ell_{-}(t)=\ell_{-}(0) e^{-\lambda t}$ of the dimension in the other direction. The area $A \simeq \ell_{+} \ell_{-}$is 
then time independent Initially, $\ell_{ \pm}(0)<1$ The constriction at the superconductor acts as a bottleneck, enforcing $\ell_{ \pm}(T)<1$ These two inequalities imply $\ell_{+}(t)<e^{\lambda(t-T)}$, $\ell_{-}<e^{-\lambda t}$ The enclosed area, therefore, has the upper bound

$$
A_{\max } \simeq p_{F} W e^{-\lambda T} \simeq \hbar N e^{-\lambda T},
$$

whete $N \simeq p_{F} W / \hbar \gg 1$ is the number of channels in the point contact

We now contınue with the quantization The two invar lants $\varepsilon$ and $T$ define a two-dimensional tor us in the fout-dimensional phase space Quantization of this adiabatically invariant torus proceeds following Einstein Bi1llouın-Kelle1 [3], by quantizing the area

$$
\oint p d q=2 \pi \hbar(m+\nu / 4), \quad m=0,1,2
$$

enclosed by each of the two topologically independent contours on the tor us Equation (4) ensures that the wave functions aie single valued (See Ref [15] for a deilvation in a two-sheeted phase space) The integer $\nu$ counts the number of caustics (Maslov index) and in our case should also include the number of Andieev reflections

The first contour follows the quasiperiodic orbit of Eq (2), leading to

$$
\varepsilon T=\left(m+\frac{1}{2}\right) \pi \hbar, \quad m=0,1,2
$$

The quantization condition (5) is sufficient to deteimine the smoothed density of states $\rho(\varepsilon)$, using the classical piobability distisbution $P(T) \propto \exp (-T N \delta / h)$ [16] for the time between Andieev reflections (We denote by $\delta$ the level spacing in the isolated billaid) The density of states

$$
\rho(\varepsilon)=N \int_{0}^{\infty} d T P(T) \sum_{m=0}^{\infty} \delta\left[\varepsilon-\left(m+\frac{1}{2}\right) \pi \hbar / T\right]
$$

has no gap but vanishes smoothly $\propto \exp (-N \delta / 4 \varepsilon)$ at energies below the Thouless energy $N \delta$ This "BohtSommer feld appioxımation" [12] has been quite successful [17-19], but it gives no information on the location of individual energy level - nor can it be used to determine the wave functions

To find these we need a second quantization condition, which is provided by the area $\oint_{T} p_{\lambda} d x$ enclosed by the contouis of constant $T\left(x, p_{x}\right)$,

$$
\oint_{T} p_{\imath} d \lambda=2 \pi \hbar(n+\nu / 4), \quad n=0,1,2,
$$

Equation (7) amounts to a quantization of the peisod $T$, which together with $\mathrm{Eq}$ (5) leads to a quantization of $\varepsilon$ For each $T_{n}$ there is a ladder of Andreev levels $\varepsilon_{n m}=\left(m+\frac{1}{2}\right) \pi \hbar / T_{n}$

While the classical $T$ can become arbitiaisly laige, the quantized $T_{n}$ has a cutoff The cutoff follows fiom the maximal area (3) enclosed by an isochionous contour
Since $\mathrm{Eq}$ (7) requires $A_{\max }>2 \pi \hbar$, we find that the longest quantized period is $T_{0}=\lambda^{-1}[\ln N+\mathcal{O}(1)]$ The lowest Andieev level associated with an adiabatically invariant tor us is therefore

$$
\varepsilon_{00}=\frac{\pi \hbar}{2 T_{0}}=\frac{\pi \hbar \lambda}{2 \ln N}
$$

The time scale $T_{0} \propto|\ln \hbar|$ repiesents the Ehienfest time of the Andieev billiaid, which sets the scale for the excitation gap in the semiclassical limit [20-22]

We now turn fiom the energy levels to the wave functions The wave function has electron and hole components $\psi_{ \pm}(x, y)$, collesponding to the two sheets of phase space By projecting the invariant tor us in a single sheet onto the $x-y$ plane we obtain the suppoit of the election or hole wave function This is shown in Fig 3, for the same billiaid presented in the pievious figuies The curves are stieamlines that follow the motion of individual electrons, all shaing the same time $T$ between Andieev reflections (A single one of these trajectories was shown in Fig 1)

Together the streamlines form a flux tube that represents the suppoit of $\psi_{+}$The width $\delta W$ of the flux tube is of order $W$ at the constinction but becomes much smaller in the inter ion of the billiard Since $\delta W / W<\ell_{+}+\ell_{\ldots}<$ $e^{\lambda(t-T)}+e^{-\lambda t}$ (with $0<t<T$ ), we conclude that the flux tube is squeezed down to a width

$$
\delta W_{\mathrm{min}} \simeq W e^{-\lambda T / 2}
$$

The flux tube for the level $\varepsilon_{00}$ has a minımal width $\delta W_{\min } \simeq W / \sqrt{N}$ Patticle conservation implies that $\left|\psi_{+}\right|^{2} \propto 1 / \delta W$, so that the squeezing of the flux tube is

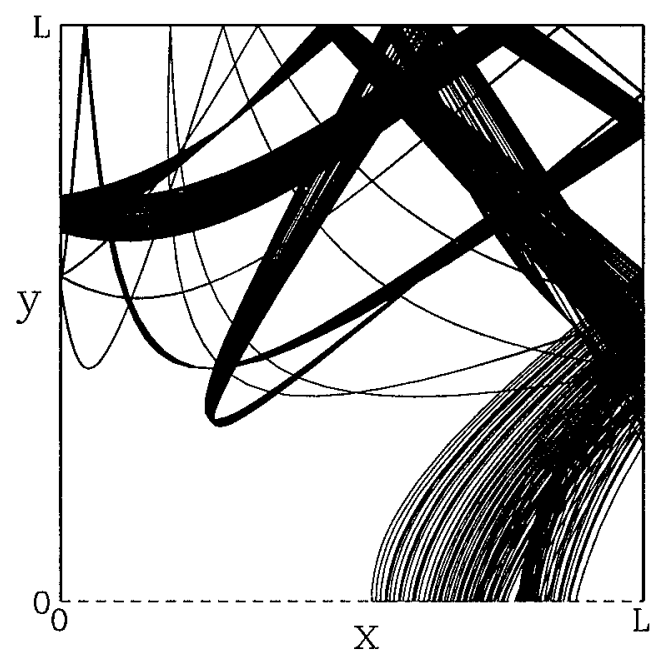

FIG 3 Projection onto the $x y$ plane of the invariant tor us with $T=18$, iepresenting the suppoit of the election compo nent of the wave function The flux tube has a laige width neat the supeiconductor which is squeezed to an indistinguishably small value after a few collisions with the boundailes 
associated with an increase of the election density by a factor of $\sqrt{N}$ as one moves away fiom the constriction

Let us examine the range of validity of adiabatic quantization The diff $\delta x, \delta p_{\lambda}$ upon one iteration of the Poincare map should be small compared to $W p_{\Gamma}$. We estimate

$$
\frac{\delta x}{W} \simeq \frac{\delta p_{\mathrm{r}}}{p_{F}} \simeq \frac{\varepsilon_{n m}}{\hbar \lambda N} e^{\lambda T_{1}} \simeq\left(m+\frac{1}{2}\right) \frac{e^{-\lambda\left(T_{0}-T\right)}}{\lambda T_{n}}
$$

For low-lying levels $(m \sim 1)$ the dimensionless disft is $\ll 1$ for $T_{n}<T_{0}$ Even for $T_{n}=T_{0}$ one has $\delta x / W \simeq$ $1 / \ln N \ll 1$

Semiclassical methods allow one to quantize only the trajectories with perlods $T \leq T_{0}$ The part of phase space with longel periods can be quantized by 1 andom-matiı theory, accolding to which the excitation gap $E_{\mathrm{gdp}}$ is the inverse of the mean time between Andreev reflections in that part of phase space $[12,17]$

$$
E_{\text {gap }}=\gamma^{5 / 2} \hbar \frac{\int_{T_{0}}^{\infty} P(T) d T}{\int_{T_{0}}^{\infty} T P(T) d T}=\frac{\gamma^{5 / 2} \hbar}{T_{0}+2 \pi \hbar / N \delta}
$$

Heie $\gamma=\frac{1}{2}(\sqrt{5}-1)$ is the golden 1atio This formula describes the crossove1 from $E_{\text {gap }}=\gamma^{5 / 2} \hbar / T_{0}=$ $\gamma^{5 / 2} \hbar \lambda / \ln N$ to $E_{\text {gap }}=\gamma^{5 / 2} N \delta / 2 \pi$ at $N \ln N \simeq \hbar \lambda / \delta$ It requires $\hbar \lambda / N \delta \gg 1$ (mean dwell time laige compated to the Lyapunov time) The semiclassical (large- $N$ ) limit of Eq (11), $\lim _{N \rightarrow \infty} E_{\text {gap }}=030 \hbar / T_{0}$ is a factor of 5 below the lowest adiabatic level, $\varepsilon_{00}=$ $16 \hbar / T_{0}$, so that indeed the energy tange neai the gap is not accessible by adiabatic quantization [23]

Up to now we considered two-dimensional Andieev billiaids Adrabatic quantization may equally well be applied to thiee-dimensional systems, with the area enclosed by an isochionous contour as the second adiabatic invariant For a fully chaotic phase space with two Lyapunov exponents $\lambda_{1}, \lambda_{2}$, the longest quantized period is $T_{0}=\frac{1}{2}\left(\lambda_{1}+\lambda_{2}\right)^{-1} \ln N$ We expect interesting quantum size effects on the classical localization of Andieev levels discovered in Ref [7], which should be measurable in a thin metal film on a superconducting substiate

One important challenge for future research is to test the adiabatic quantization of Andreev levels numerically, by solving the Bogoliubov-de Gennes equation on a computer The characteristic signature of the adiabatic invailant that we have discovered, a nallow region of enhanced intensity in a chaotic iegion that is squeezed as one moves away from the superconductor, should be readily observable and distingurshable from other features that are unielated to the presence of the superconductor, such as scars of unstable periodic orbits [10] Expe11mentally these regions might be obser vable using a scanning tunneling probe, which provides an eneigy and spatially resolved measurement of the election density

This work was suppoited by the Dutch Science Foundation NWO/FOM We thank I Adagideli and
J Twor zydło for helpful discussions

[1] P Ehrenfest, Ann Phys (Leip7ıg) 51, 327 (1916)

[2] C C Martens, R L Waterland, and W P Reinhardt, J Chem Phys 90, 2328 (1989)

[3] M C Gutzwillei, Chaos in Classical and Quantum Mechanics (Spı ingel, Berlın, 1990)

[4] R E Prange, in The Quantum Hall Effect, edited by $\mathrm{R} E$ Prange and $\mathrm{S} M$ Girvin (Springer, New Yolk, 1990)

[5] I Kosztin, D L Maslov, and P M Goldbait, Phys Rev Lett 75, 1735 (1995)

[6] M Stone, Phys Rev B 54, 13222 (1996)

[7] A V Shytov, P A Lee, and L S Levitov, Phys Usp 41, 207 (1998)

[8] I Adagidelt and P M Goldbait, Phys Rev B 65, 201306 (2002)

[9] J Wlersig, Phys Rev E 65, 036221 (2002)

[10] E J Heller, Phys Rev Lett. 53, 1515 (1984)

[11] PW O'Conno1, J Gehlen, and E Hellel, Phys Rev Lett 58, 1296 (1987)

[12] J A Melsen, PW Biouwer, K M Frahm, and C W J Beenakkeı, Euıophys Lell 35, 7 (1996)

[13] Isochronous contours are defined as $T\left(\lambda, p_{\lambda}\right)=$ const at $\varepsilon=0$ We assume that the isochronous contous are closed This is tiue it the border $p_{3}=0$ of the classically allowed region in the $\lambda, p$, section is itself an 1sochionous contoul, which is the case if $\lim _{1 \rightarrow 0} \partial V / \partial y \leq 0$ In this case the pattucle leaving the superconductor with infinitesimal $p$, cannot penetiate into the billiaid

[14] Adrabatic invailance is defined in the limit $\varepsilon \rightarrow 0$ and is therefore distinct from invaliance in the sense of Kolmogorov-Ainold Moser (KAM), which would ie quic a cilical $\varepsilon$ such that a contour is exactly invairant for $\varepsilon<\varepsilon^{4}$ Numerical evidence [5] suggests that the KAM theorem does not apply to a chaotic Andreev billard

[15] K P Duncan and B L Gyo1ffy, Ann Phys (NY) 298, 273 (2002)

[16] W Baue and G F Bertsch, Phys Rev Lett, 65, 2213 (1990)

[17] H Schomerus and C W J Beenakkel, Phys Rev Lett 82, 2951 (1999)

[18] W Ihia, M Leadbeater, J L Vega, and K Richtel, Europhys J B 21, 425 (2001)

[19] J Cseit1, A Kormányos, Z Kaufmann, J Kolta1, and C J Lambert, Phys Rev Lett 89, 057001 (2002)

[20] A Lodder and Yu V Nazarov, Phys Rev B 58, 5783 (1998)

[21] D Taras-Semchuk and A Altland, Phys Rev B 64, $014512(2001)$

[22] I Adagidelı and C W J Beenakker, Phys Rev Lett 89, 237002 (2002)

[23] The density of states near the gap is obtained in the same way as Eq (11), with the result $\rho(\varepsilon)=c\left(\varepsilon-E_{\mathrm{glp}}\right)^{1 / 2} \times$ $N_{\mathrm{ufl}}^{1 / 2} \delta_{\mathrm{eff}}^{3 / 2}, \quad$ where $N_{\mathrm{etf}}=N^{1-N \delta / h \lambda}, \quad \delta_{\text {eff }}^{-1}=\left(\delta^{-1}+\right.$ $N \ln N / h \lambda) N^{-N \delta / h \lambda}$, and $c=4(\pi / \sqrt{5})^{1 / 2} \gamma^{5 / 4}(9+$ $4 \sqrt{5})^{2 / 3} \approx 18$ 